\title{
Fine Epitope Mapping of the Human SS-B/La Protein Identification of a Distinct Autoepitope Homologous to a Viral Gag Polyprotein
}

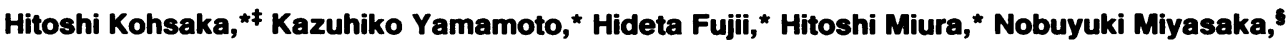 \\ Kusuki Nishioka," and Terumasa Miyamoto* \\ ${ }^{*}$ Department of Medicine and Physical Therapy, Faculty of Medicine, University of Tokyo, Tokyo, 113, Japan; ${ }^{\ddagger}$ The First Department \\ of Internal Medicine, and \$ Department of Virology and Immunology, Medical Research Institute, Tokyo Medical and Dental \\ University, Tokyo, 113, Japan; and "Institute of Rheumatology, Tokyo Women's Medical College, Tokyo, 163, Japan
}

\begin{abstract}
To analyze the autoepitopes on the SS-B/La protein, a cDNA covering the entire region coding the protein was isolated from a human cDNA library. The cDNA was subcloned into an expression plasmid vector, $\mathrm{pEX}$, to express its protein product as a fusion protein with cro- $\beta$-galactosidase in Escherichia coli. $A$ recombinant $\mathrm{pEX}$ plasmid expressing three-fourths of the protein (amino acid 112-408) was also constructed. The antigenicities of these recombinant proteins were confirmed with a patient's serum. Their various deletion mutants were produced with exonuclease III treatment from the $3^{\prime}$ ends of the cDNAs without changing the proper translational frame. Immunoblot analysis and enzyme-linked immunosorbent assay were used to evaluate the reactivities of the recombinant proteins with patients' sera to determine the autoepitopes. A narrow segment (amino acid 88-101) and the region where several epitopes were located (amino acid 283-338) on the SS-B/La protein were universally recognized by all the sera with anti-SS-B/La antibodies examined. An additional epitope region (amino acid 179-220) was recognized by some patients' sera. Computer analysis revealed that the most distinct autoepitope, amino acid 88-101, had a striking homology to a retroviral gag polyprotein. These findings indicate that exogenous or endogenous retroviruses may play a role in initiation of the anti-SS-B/La autoimmunity. (J. Clin. Invest. 1990. 85:1566-1574.) autoantibody $\bullet$ autoantigen $\bullet$ anti-SS-B/La antibody $\bullet$ molecular mimicry • Sjögren's syndrome
\end{abstract}

\section{Introduction}

Autoantibodies directed against various nuclear proteins of normal cells are detectable in sera from patients with systemic autoimmune diseases $(1,2)$. Although it seems unlikely that these antinuclear antibodies directly injure the organs, some antibodies are related with particular diseases or symptoms. Analysis of the mechanisms of autoantibody production should therefore provide important clues to the pathogenesis of autoimmune disorders. Several mechanisms of autoantibody production have been proposed by previous investiga-

Address reprint requests to Dr. Yamamoto, Department of Medicine and Physical Therapy, Faculty of Medicine, University of Tokyo, 7-3-1 Hongo, Bunkyo-ku, Tokyo 113, Japan.

Received for publication 6 October 1989 and in revised form 29 December 1989.

J. Clin. Invest.

(C) The American Society for Clinical Investigation, Inc. 0021-9738/90/05/1566/09 \$2.00

Volume 85, May 1990, 1566-1574 tors. They include polyclonal B cell activation (3), an antigendriven mechanism (4) and molecular mimicry (5). To determine which mechanism is predominantly involved in the production of a particular autoantibody, it is useful to analyze the numbers and region of autoepitopes recognized by patients' sera.

The SS-B/La antigen is a small nuclear ribonucleoprotein $(\mathrm{snRNP})^{1}$ with a molecular weight of $47 \mathrm{kD}$. Anti-SS-B/La antibodies are strongly associated with primary Sjögren's syndrome (SS), and serve as a diagnostic marker of SS. The SS-B/ $\mathrm{La}$ protein is mainly associated with the precursor forms of RNA polymerase III transcripts, including transfer RNAs, 7S RNA, 5S RNA (7), and virus-encoded RNAs such as adenovirus-encoded RNAs (VAI and VAII) (8), Epstein-Bar virus-encoded RNAs (EBER) (9) and leader RNAs of some negative stranded viruses $(10,11)$. Recent studies have shown that the SS-B/La protein is essential in the biogenesis of these RNAs (7, 12). However, it remains to be clarified why autoantibodies against such an important molecule are related to SS, an autocrine exocrinopathy.

Several investigators have performed epitope mapping of the autoepitopes on the SS-B/La protein (13-17). However, their results were not able to lead to clear explanation of autoantibody production, since they were only assigned in long stretches of peptide. We report here the isolation of a fulllength cDNA encoding the human SS-B/La autoantigen and its epitope mapping with deletion mutants, which revealed three antigenic regions. The most distinct and universally recognized epitope was found to be homologous to a retroviral gag polyprotein. These results raise the possibility that molecular mimicry might induce anti-SS-B/La autoimmunity.

\section{Methods}

Patient sera. Sera were obtained from patients with SS or other autoimmune diseases who were treated in the hospitals of the University of Tokyo, Tokyo Medical and Dental University, and Kanazawa Medical College. Precipitating antibodies against the SS-B/La protein were evaluated with the double immunodiffusion method using rabbit thymus extract and a standard anti-SS/La serum.

Isolation of CDNAs for the human SS-B/La protein from $\lambda g t 11$ expression library and pcD library. Details of isolation have been described previously $(18,19)$. Following the procedures of Young and Davis (20), a human fibroblast cDNA expression library constructed in the $\lambda$ gt 11 vector was screened with serum from a patient with primary SS, containing anti-SS-B/La antibodies as an antibody probe. After screening with immunostaining, each cDNA insert of positive clones was subcloned into an expression plasmid pEX-2 (21) as described in

1. Abbreviations used in this paper: ExoIII, exonuclease III; snRNP, small nuclear ribonucleoprotein; SS, Sjögren's syndrome. 
the next paragraph. The recombinant pEXs were induced to express fusion proteins with cro- $\beta$-galactosidase and products were evaluated by their reactions to the patient's serum. The longest insert among positive clones was named pA158 and its characteristics as a part of cDNA for the human SS-B/La protein were reported (19). For the isolation of full-length $\mathrm{cDNAs}$, the screening of $\mathrm{pcD}$ plasmid libraries (22) constructed from fibroblasts and foreskin cells was performed with ${ }^{32} \mathrm{P}$-labeled pA158 cDNA as a hybridization probe. The longest cDNA insert among positive clones was derived from the fibroblast library and verified as the full-length cDNA for the human SS-B/La protein.

Expression of cloned cDNA. To express the cloned cDNAs, cDNA inserts were recovered from vectors with restriction enzyme digestion (Eco RI for $\lambda \mathrm{gt} 11$, Stu I and Bam HI for pcD plasmid) and subcloned into expression vector $\mathrm{pEX}$ plasmids at their polylinkers. To adjust the translational frame, three $\mathrm{pEXs}$ with different frames $(\mathrm{pEX}-1,2,3)$ were used for subcloning. Escherichia coli, POP2136 transformed by recombinant pEX, were grown in a Luria-Bertani medium overnight at $30^{\circ} \mathrm{C}$. The cultures were then shifted to $42^{\circ} \mathrm{C}$ and incubated for $2 \mathrm{~h}$ to express the fusion proteins with cro- $\beta$-galactosidase. Recombinant pEXs with a correct frame were selected by examining the reactivities of their products with the patient's serum.

Affinity-purification of autoantibodies. A modified method of Olmsted (23) was used for purification of autoantibodies. Bacterial lysates with induced recombinant autoantigens were electrophoresed on polyacrylamide/SDS gels and transferred to nitrocellulose membranes. After staining with Ponceau S solution (Sigma Chemical Co., St. Louis, MO), the protein bands expected to contain fusion proteins were cut out and these pieces of membrane were saturated with PBS-0.5\% skim milk (Yukijirushi, Tokyo, Japan) followed by $0.5 \%$ Tween 20 in PBS for $30 \mathrm{~min}$. They were then incubated with a patient's serum diluted $1: 10$ in PBS-0.5\% skim milk followed by washing five times with PBS. The bound antibodies were eluted with $4 \mathrm{ml}$ of 0.2 $\mathrm{M}$ glycine- $\mathrm{HCl}, \mathrm{pH} 2.6$, containing $0.15 \mathrm{M} \mathrm{NaCl}$ and $1 \% \mathrm{BSA}$. After 5 min of incubation, the eluate was neutralized with $1 \mathrm{ml}$ of $1 \mathrm{M}$ Tris$\mathrm{HCl}, \mathrm{pH} 7.5$.

Immunoblotting. The procedures were reported previously in detail (18). The lysates of human peripheral leukocytes or bacteria with induced proteins were separated on polyacrylamide/SDS gels and transferred to nitrocellulose membranes. These were reacted with sera or affinity-purified antibodies. The antigenic protein bands were detected with rabbit anti-human IgG antibodies then goat anti-rabbit immunoglobulin antibodies coupled with horseradish peroxidase.

Construction of expression plasmids with deleted cDNAs. To construct a cDNA lacking one-fourth of the $5^{\prime}$ terminal (this cDNA is hereafter referred to as FSBB), a full-length cDNA (referred to as FSBF) was cut with the authentic Bgl II site and its $3^{\prime}$ part was subcloned into $\mathrm{pEX}$ plasmids in three different translational frames. The recombinant $\mathrm{pEX}$ with the correct frame was selected using the patient's serum. Restriction enzymes and exonuclease III (ExolII; Takara, Kyoto, Japan) were used for the modification and deletion of the 3 ' terminal of the cDNAs, essentially by the methods of Henikoff (24) and Putney (25). The plasmids with full-length cDNAs were digested with restriction enzyme Sal I and filled in $3^{\prime}$ recessed ends with alphaphosphorothioate deoxynucleotides (Promega Biotek, Madison, WI) and Klenow DNA polymerase (Takara). Thereafter, they were digested with restriction enzyme Bam HI. The other plasmid, FSBB, was digested with Pst I and Bam HI. After deletion with ExoIII, end repair with mung bean nuclease (Takara) and Klenow DNA polymerase was performed before ligation with T4 DNA ligase (Takara). E. coli hosts were transformed with the ligated vectors. Plasmids containing cDNAs of different length, were obtained by changing the duration of ExoIII treatment.

DNA sequencing. The isolated cDNA inserts and deleted inserts were subcloned into bluescript plasmid vectors (Strategene, La Jolla, CA) to be sequenced. Their sequences were determined by Sanger's dideoxy method (26) using modified T7 DNA polymerase (USB, Cleveland, $\mathrm{OH}$ ). To sequence the full-length cDNA in bluescripts, they were also deleted with ExoIII. The sequences of DNAs and deduced amino acids were analyzed using SDC-GENETYX Genetic Information Processing Software (Software Developing Co., Tokyo, Japan), the protein sequence database of the National Biomedical Research Foundation (NBRF) and SWISS-PROT protein sequence database of European Molecular Biology Laboratory (EMBL).

Enzyme-linked immunosorbent assay (ELISA). After induction of the fusion protein in $E$. coli, bacteria were harvested by centrifugation from $500-\mathrm{ml}$ cultures and lysed in $20 \mathrm{ml}$ of lysozyme solution [1 $\mathrm{mg} / \mathrm{ml}$ Lysozyme (Wako, Osaka, Japan), $8 \%$ sucrose, $5 \%$ Triton $\mathrm{X}-100,50 \mathrm{mM}$ EDTA, $50 \mathrm{mM}$ Tris- $\mathrm{HCl}, \mathrm{pH}$ 8.0] with sonication. After centrifugation, pellets were suspended in $20 \mathrm{ml}$ of DNase buffer [5 g/ml DNase I (Sigma Chemical Co.)], $150 \mathrm{mM} \mathrm{NaCl}, 10 \mathrm{mM}$ $\mathrm{MnCl}_{2}, 50 \mathrm{mM}$ Tris-HCl, $\mathrm{pH} \mathrm{7.6]} \mathrm{for} 2 \mathrm{~h}$ at $37^{\circ} \mathrm{C}$ followed by sonication to fragmentate the bacterial DNAs. After centrifugation, the pellets were washed twice with $20 \mathrm{ml}$ of the solution containing $0.5 \%$ Triton X-100, $10 \mathrm{mM}$ EDTA, $50 \mathrm{mM} \mathrm{NaCl}$ and $50 \mathrm{mM}$ Tris- $\mathrm{HCl}$, pH 7.6. Finally, the purified pellets were solubilized by adding $4 \mathrm{ml}$ of $8 \mathrm{M}$ urea and $10 \mathrm{mM}$ DTT in PBS. Each well of multititer plates was coated with $3 \mu \mathrm{g} / 150 \mu \mathrm{l}$ of the purified protein in carbonate buffer, $\mathrm{pH} 9.6$, followed by blocking with $0.5 \%$ skim milk and $0.05 \%$ Tween 20 in PBS. Human sera were preincubated with bacterial lysate containing nonrecombinant pEX products. Serially diluted sera with $1 \%$ skim milk in PBS and, subsequently, peroxidase-coupled protein A were applied to the plate. Bound antibodies were detected enzymatically with $o$-phenylenediamine as the substrate, using a microplate-photometer at $492 \mathrm{~nm}$. The quantity of coated proteins was evaluated by applying rabbit anti- $\beta$-galactosidase. Binding units defined as the reciprocal of the serum dilution that produced an $\mathrm{OD}_{492}$ of 1.0 were used to quantitate reactivity.

\section{Results}

Isolation of full-length cDNAs encoding the human SS-B/La protein. Using serum from a patient (T.S.) which contains precipitating antibodies against the SS-B/La protein, $\lambda g t 11$ human fibroblast cDNA expression library was screened. The longest CDNA insert $(1.5 \mathrm{~kb})$ was named pA158 clone and analyses of its product in pEX expression vector was previously reported (19). To obtain a full-length $\mathrm{CDNA}$, pcD plasmid libraries constructed from human fibroblasts and foreskin cells were screened with labeled pA158 cDNA. After screening of each library, the longest cDNA $(1.8 \mathrm{~kb})$ was derived from fibroblast library and named FSBF clone. This was subcloned into $\mathrm{pEX}$ plasmids, and a recombinant clone with a correct translational frame was selected by immunostaining with the patient's serum. The protein induced using FSBF (named FSBF. EX) and the vector-produced protein (pEX) were separated by $8 \%$ SDS-polyacrylamide gel electrophoresis (PAGE) and electroblotted to nitrocellulose membranes. The anti-pEX or anti-FSBF. EX antibodies was purified by affinity-purification with these membranes. In the immunoblot analysis of human peripheral leukocyte proteins fractionated in $12.5 \%$ SDS-PAGE, a standard anti-SS-B/La serum (a serum from patient T.S.) and anti-FSBF-EX antibodies recognized an identical polypeptide with a molecular weight of $\sim 50 \mathrm{kD}$ (Fig. 1). For further characterization, human peripheral leukocyte lysate was incubated and precipitated with antiFSBF - EX antibodies. After phenol extraction of the precipitates, the remaining nucleic acids were fractionated in ureaPAGE. Serum from the patient and anti-FSBF. EX antibodies precipitated RNAs that are known to be associated with the SS-B/La protein, e.g., precursors of 5S RNA and transfer RNAs (data not shown). The nucleotide sequence of FSBF cDNA determined by Sanger's method matched the pre- 


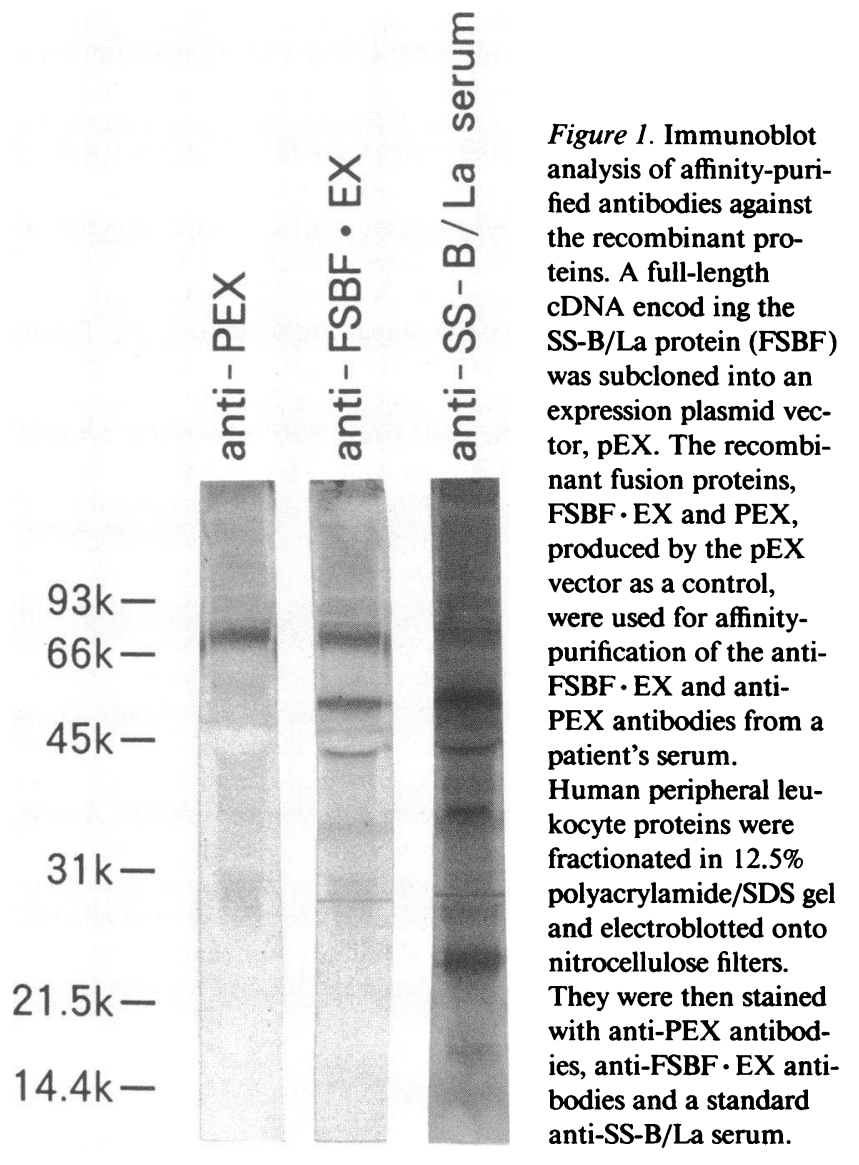

viously published one $(17,27)$. It begins -2 bp from the initiation codon and covers the entire region coding the SS-B/La protein.

Fine epitope mapping of the $S S-B / L$ a protein using deleted $c D N A s$. To identify the autoepitopes on the human SS-B/La protein, the recombinant $\mathrm{pEX}$ plasmid with FSBF cDNA was deleted enzymatically from the $3^{\prime}$ end of the cDNA fragment by ExoIII treatment. Because this procedure does not change the proper translational frames of the cro- $\beta$-galactosidase gene and the inserted cDNAs, the resultant deletion mutants are capable of expressing the truncated proteins in the correct translational frame. The inserts of the representative deletion mutants (FD1-FD6) used for epitope mapping are shown in Fig. 2. They were all induced to express the truncated SS-B/La autoantigens as fusion proteins in bacteria, and the products were separated on polyacrylamide/SDS gels (Fig. $3 A$ ). Staining with Coomassie brilliant blue showed the recombinant products were equally large in amount and their molecular weights depended on the length of CDNA inserts. Their reactivity with sera containing anti-SS-B/La antibodies was determined by immunostaining. As shown in Fig. $3 A$ and Table I, the most intensive binding of antibodies was found in FSBF. In all sera examined, reactivities dropped dramatically between the FD5 and FD6 products. FD5 reacted less than FD4. These findings indicated that one of the major autoepitopes is located in the area between the $\mathrm{COOH}$-terminals of the FD4 and FD6 products (named Ept1, Fig. 4). Moreover, since the difference between FD5 and FD4 is only four amino acids, this epitope seems to reside in the vicinity of the $\mathrm{COOH}$-terminal of the FD5 product.
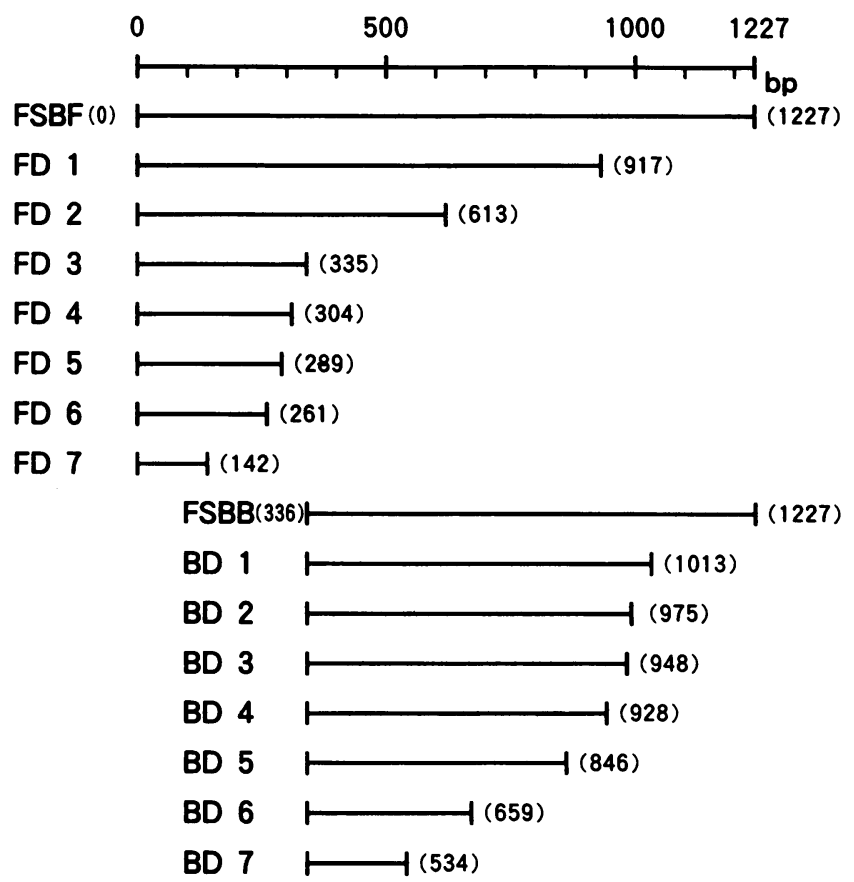

Figure 2. Schematic diagram of a full-length cDNA (FSBF) encoding the SS-B/La protein and its deletion mutants used for immunoblot analysis and ELISA. FSBF was enzymatically manipulated to make deletion mutants FD1-FD7 with exonuclease III, and FSBB was constructed using $\mathrm{Bg} 1 \mathrm{II}$ restriction enzyme. FSBB was also deleted to make BD1-BD7. The pEX expression vectors containing them were induced to express hybrid proteins with cro- $\beta$-galactosidase.

That the reactivity of FSBF was superior to those of FD1 and FD2 in most sera suggested the existence of another distinct autoepitope at the $\mathrm{COOH}$-terminal portion of the SS-B/ La protein. Therefore, to exclude the influence of Ept 1 antigenicity, the FSBF cDNA insert was cut at the Bgl II restriction site ( $335 \mathrm{bp}$ from the initiation codon), and its $3^{\prime}$ part (named FSBB) was subcloned into $\mathrm{pEX}$ vectors. The recombinant $\mathrm{pEX}$ with FSBB in the proper translational frame was selected by the same serum. As expected, its product was found to react with all the sera containing anti-SS-B/La autoantibodies. To localize other epitopes, deletion mutants (BD1-BD7), derived from FSBB, were generated in the same way as FSBF (Fig. 2). The reactivities of the products with patients' sera were also examined (Fig. $3 B$ and Table I). In all sera, decreased reactivity was mainly found from BD1 to BD5. This area between the $\mathrm{COOH}$-terminal of $\mathrm{BD} 1$ and BD5 products was named Ept 2 (Fig. 4). However, there were no common segments where the intensities of staining changed among the eleven sera tested. The change was found between BD1 and BD2 in sera from patients 2,10 , and 11 . The reactivity in sera from the patients 8,10 , and 11 was altered between BD2 and BD3; in sera from patients $1-9$, and 11 between BD3 and BD4; and in sera from patients 1, 3, 5-7, and 9 between BD4 and BD5. Each serum had more than two regions that changed in intensity of staining, but nevertheless, we could not identify any distinct area in this region that was recognized by all the sera. Sera from patients 2, 4-9, and 10 reacted with BD5 and BD6 products. The intensity of staining with BD5 and BD6 products was equal in every serum and no serum reacted with BD7. This supported the presence of the third epitope region. 

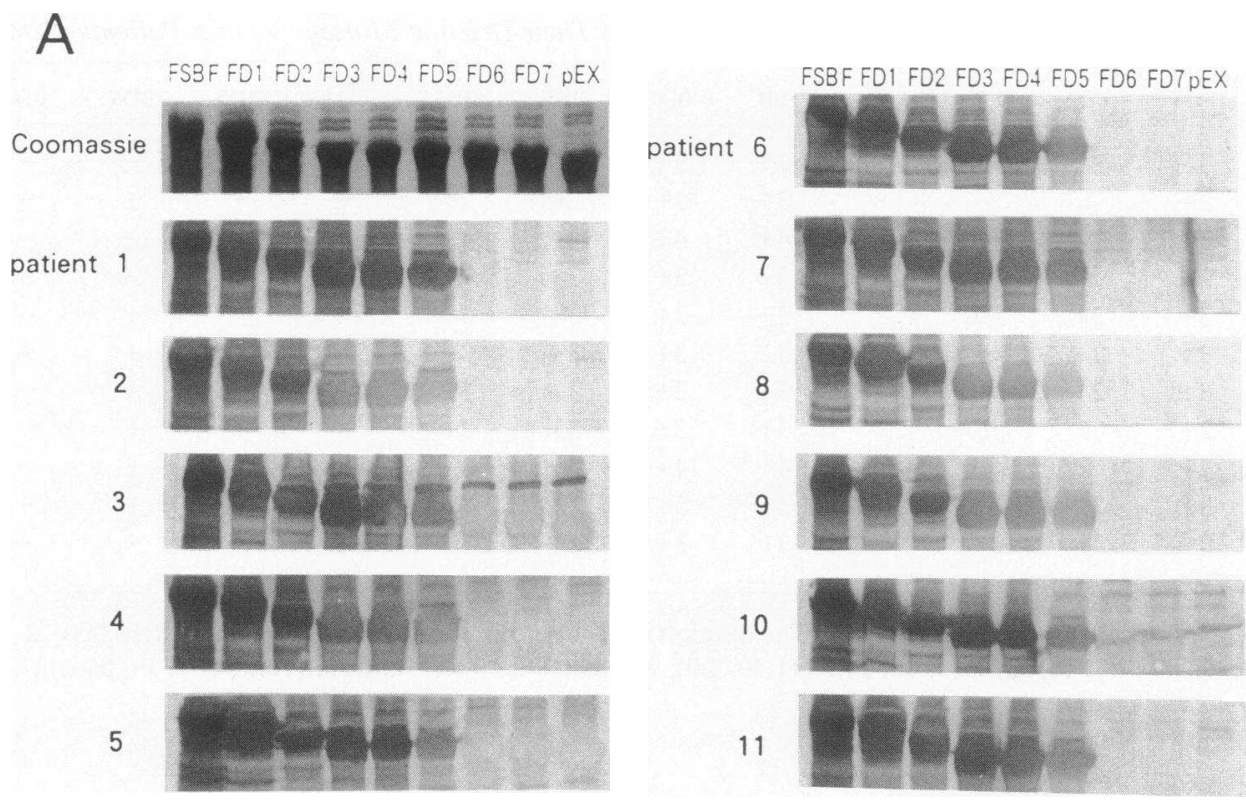

7

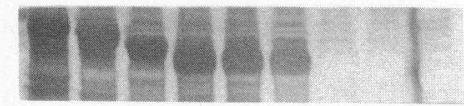

8

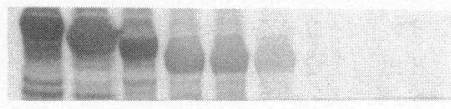

9

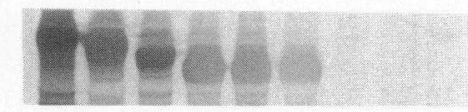

10

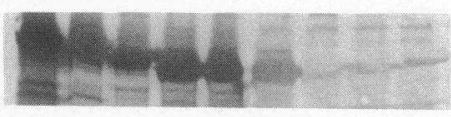

11

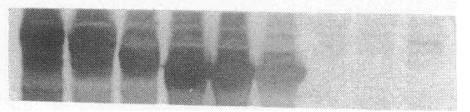

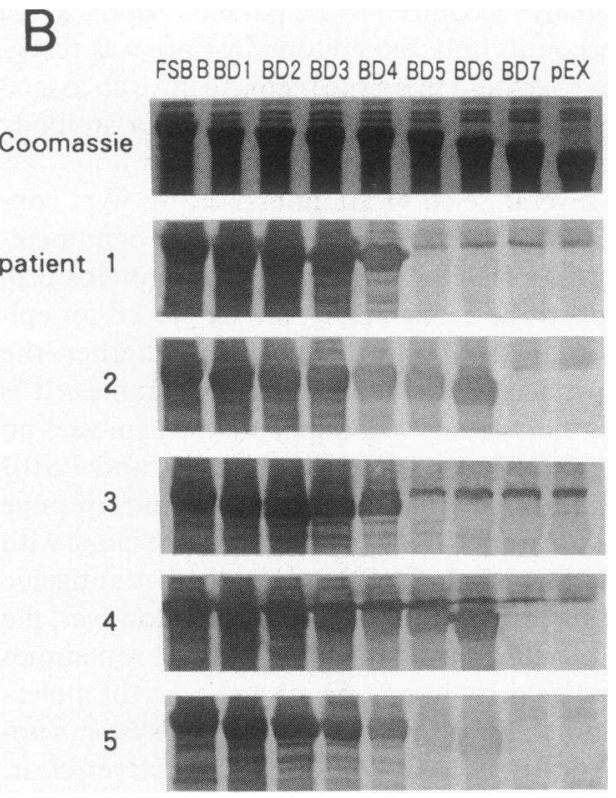

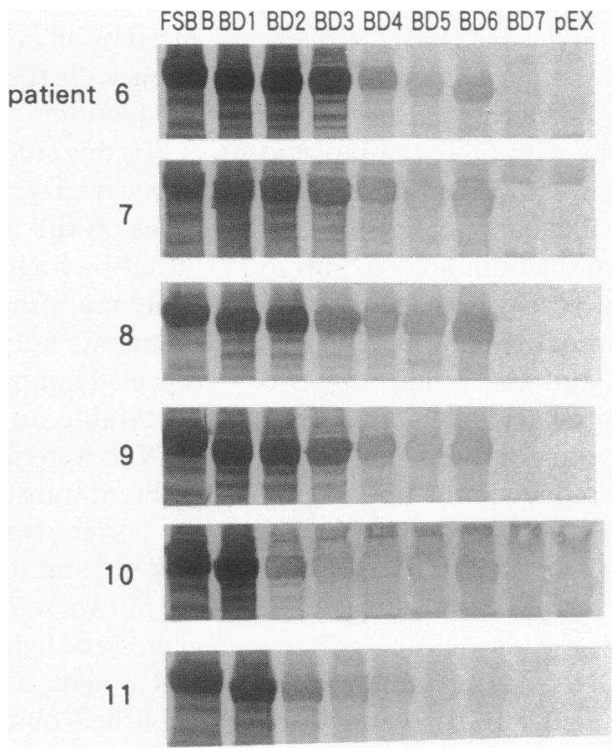

Figure 3. Antigenicities of the protein encoded by FSBF and its deletion mutants. The recombinant autoantigens ( $A$ : FSBF, FD1-FD7; $B$ : FSBB, BD1-BD7) and the vectorproduced protein ( $\mathrm{pEX}$ ) were separated in $8 \%$ polyacrylamide/SDS gels. One of each was stained with Coomassie brilliant blue and the others were transferred onto nitrocellulose filters. Immunostaining with 11 sera from patients containing anti-SS-B/La antibodies was performed.

We named the peptide between the $\mathrm{COOH}$-terminals of BD6 and BD7 products Ept 3 but it was recognized only by some of the patients' sera.

ELISA using deletion mutants. To substantiate that all sera from patients with anti-SS-B/La autoantibody predominantly react with Ept 1 and Ept2, sera from patients with SS or other systemic autoimmune diseases containing anti-SS-B/La precipitating antibodies were assayed using the products FD4, FD6, BD1, and BD5 and non-recombinant pEX as a control. Wells of multititer plates were coated with an equal quantity of each deleted recombinant SS-B/La protein, and patients' sera were diluted serially from $1: 100$ to $1: 3^{7} \times 100$. Assays were performed in duplicate, and in order to eliminate the effect of antibodies against bacterial components or induced $\beta$-galactosidase, the final data were obtained by subtraction of values against the $\mathrm{pEX}$ protein from those against the recombinant proteins. Two representative examples of the quantitation of antibodies against them are shown in Fig. 5. Both showed significantly decreased reactivity between FD4 and FD6 and between BD1 and BD5. The sera shown in Fig. $5 B$ still reacted with BD5, implying this serum contains antibodies against epitopes in the Ept 3 area. We tested 28 anti-SS-B/La positive sera and the antibody binding units of each serum against each protein are shown in Table II. These data showed that all sera with anti-SS-B/La antibodies reacted intensively with FD4 and BD1, but never with FD6. Reactions displayed as less than 100 binding units indicate an eventual lack of reaction, i.e., $\mathrm{OD}_{492}$ $<0.3$ in 1:100 dilution (see Fig. 5A). Therefore, even sera from patients 3, 10, 13, 16, 22, and 24 (binding units for FD4 < 200) reacted far more intensively with FD4 than with FD6. This was confirmed using immunoblot analysis (data not shown). 22 of 28 sera were found to react with BD5 even though their 
Table I. Summary of the Antigenicities of Recombinant Proteins Produced by FSBF and Their Deletion Mutants against Patients' Sera

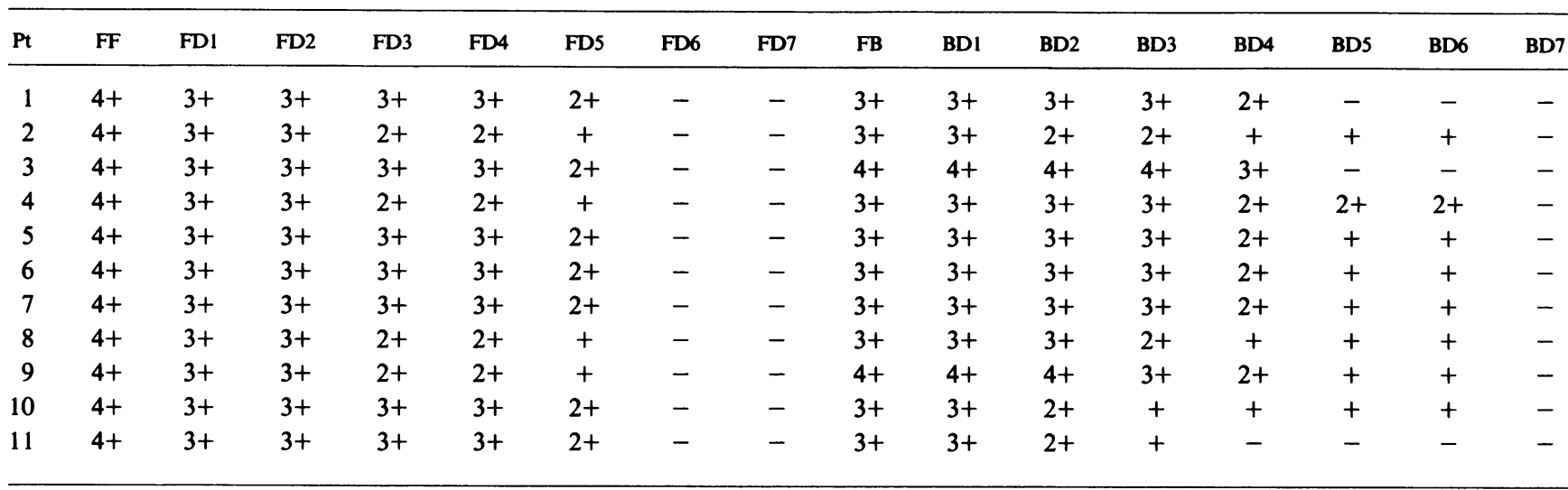

The intensity of the bound antibodies were arbitrarily graded from - to 4+. The dilution of each serum was as follows: Patient 1 (1:10,000), 2 (1:40,000), 3 (1:1,000), 4 (1:80,000), 5 (1:80,000), $6(1: 5,000), 7(1: 80,000), 8(1: 40,000), 9(1: 80,000), 10(1: 1,000), 11(1: 40,000)$ Pt, patient number, FF, FSBF, FB, FSBB.

reactivities with $\mathrm{BD} 5$ were weaker than those with $\mathrm{BD} 1$. This suggests all sera recognize predominantly the epitopes in Ept 1 and Ept2. It is also suggested that an additional autoepitope, Ept3, which is reactive with only some of sera, is located in the middle of the SS-B/La protein.

Computer analysis of the amino acid sequence of epitope regions. The amino acid sequences deduced for Ept1, Ept2, and Ept 3 were analyzed for homology with DNA/amino acid analyzing software. After comparison with other amino acid sequences in the protein sequence databases of NBRF and EMBL, Eptl was found to have a striking homology to amino acids 32 to 39 of the feline sarcoma virus gag leader protein $(28,29)$ (Fig. 6). In the COOH-terminal part of Ept1, six of eight amino acids were identical, and the remaining two amino acids and the two flanking amino acids were analogous biochemically. No homology was found for Ept2 or Ept3. There was no homology among the sequences of Ept1, Ept2, and Ept3.

\section{Discussion}

After the isolation of a full-length cDNA for the human SS-B/ La protein (FSBF), Ept 1 (amino acid 88-101), and Ept2 (amino acid 283-338) on the SS-B/La protein were found to contain distinct autoepitopes by making several deletion mutants to produce truncated proteins. Ept 1 was defined as the area between the COOH-terminals of FD4 and FD6, and Ept2 as that between the COOH-terminals of BD1 and BD5 (Fig. 4). Antibody binding with FD4, BD1 and BD5 in ELISA disclosed that all sera from patients containing anti-SS-B/La precipitating antibodies recognized both Ept 1 and Ept2. All sera that reacted potently to FD4 have no reactivity to FD6. Moreover, the intensity of antibody binding to FD5 was weaker than that to FD4, whereas the amino acid sequence difference between them is only four amino acids. These facts suggested the $\mathrm{COOH}$-terminal of FD5 might be located just on the epitope. In contrast, Ept 2 is a long stretch of peptides consisting of 56 amino acids. Although this area was reactive with all patients' sera, there seems to be no universal epitope. It is likely that Ept 1 is a single distinct autoepitope, even though not yet proved, whereas Ept 2 consists of several antigenic epitopes, the reactivities of which differ from patient to patient. In addition, not a few sera reacted with epitopes in Ept3. However, this region was not equally recognized by all patients' sera. Taking these facts into account, only the epitope in Ept 1 was recognized by all positive sera and therefore seems to have an association with the mechanisms triggering anti-SS-B/La antibody production.

In this study, several deletion mutants of FSBF were constructed enzymatically and used for the assessment of autoepitopes on the SS-B/La protein. In general, when proteins produced by fragments from a long cDNA are employed for epitope mapping, it needs to be determined whether the fragments are expressed in correct translational frames. It is especially important in the case of a fragment that expresses no detectable antigen. In our experiments, FSBF and FSBB cDNAs were first subcloned into expression plasmids in three different translational frames. As expected, only the clone with the correct translational frame produced a distinct antigenic protein, and it was selected for further analyses. However, the other two series of clones with incorrect frames sometimes expressed slight antigenicity in an unexpected range of molecular weight, a finding also observed in the $\lambda g t 11$ system (unpublished observation). The reason for this is not yet clear. Judging from the molecular weight and the amount of weakly stained recombinant protein, it could be speculated that a portion of the frame after the authentic stop codon is translated, probably because of the strong expression system in $E$. coli. To avoid making deletion mutants with an incorrect translational frame, we mainly used ExoIII treatment at the $\mathbf{3}^{\prime}$ end of the gene, and many deletion mutants were constructed without changing their frames. However, with this procedure it is difficult to determine the precise $\mathrm{NH}_{2}$-terminal boundary of the epitope and to identify the minor epitopes residing on the COOH-terminal side of the major epitopes. It is also possible that the epitope is discontinuous and the identified short peptide is a part of it. Therefore, to identify the precise range of the peptide as an autoepitope, cDNAs deleted from the $\mathrm{NH}_{2}$-terminal by other manipulations or synthetic peptides could be useful. These systems, however, do not always work properly. Thus, the method used in this study seems sufficient to determine the location of the major epitopes on the autoantigens. 


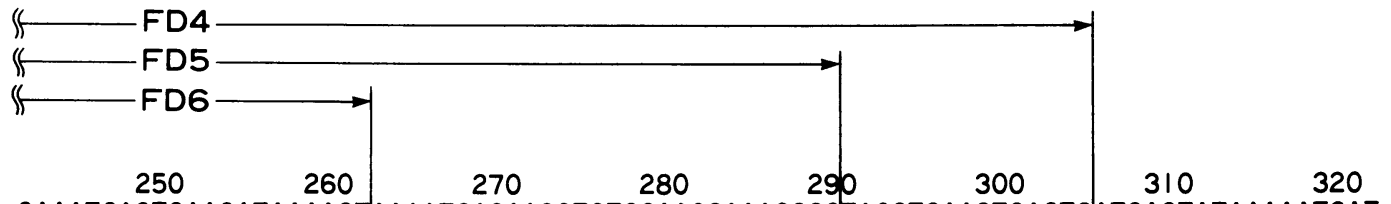

GAAATCAGTGAAGATAAAACTAAAATCAGAAGGTCTCCAAGCAAACCCOTACCTGAAGTGACTGATGAGTATAAAAATGAT

GI $u$ I I eSerGI uAspLysThrLys I I eArgArgSerProSerLysProLeuProGIuVa IThrAspGIUTyrLysAsnAsp

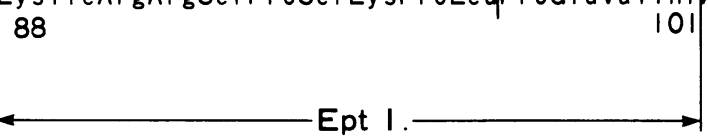

B
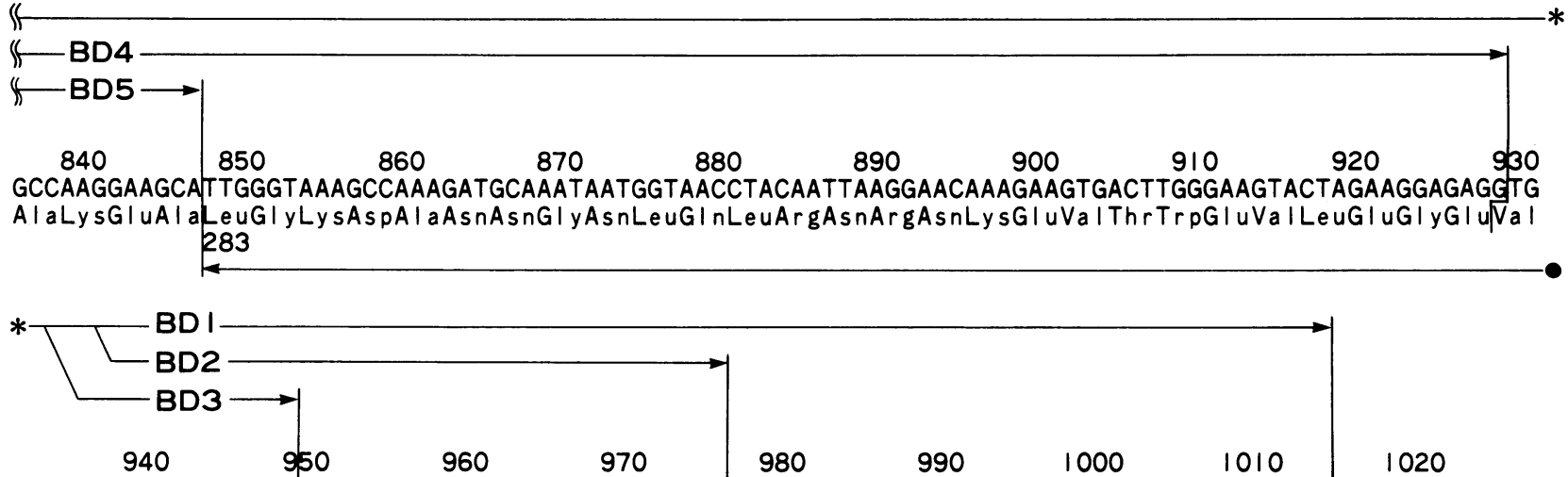

GAAAAAGAAGCACTGAAGAAAATAATAGAAGACCAACAAGAATCOCTAAACAAATGGAAGTCAAAAGGTCGTAGATTTAAAGGAAAAGGAAAGGGT

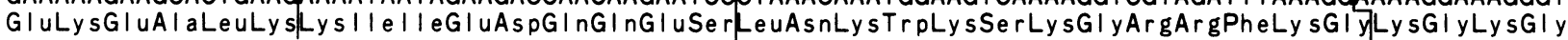

Ept 2

C
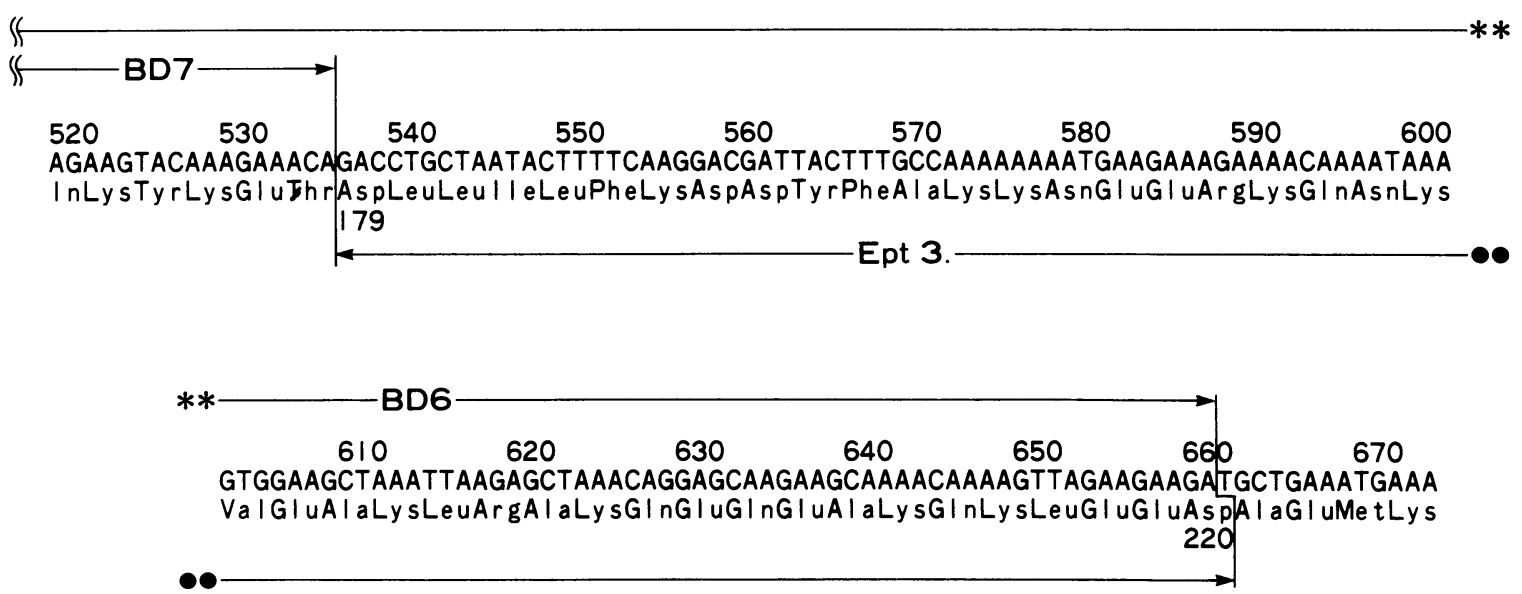

Figure 4. Nucleotide and amino acid sequences around the three autoepitope areas on the SS-B/La protein. Ept 1 is located between the COOH-terminals of FD4 and FD6. Ept 2 is between BD1 and BD5. Ept 3 is between BD6 and BD7. This figure shows the 3 ' end nucleotide sequence of each deletion mutant surrounding Ept $1(A)$, Ept2 $(B)$, and Ept3 $(C)$. The amino acids that were deduced are also shown.

The $\mathrm{pEX}$ vectors that we used have certain advantages. The pEXs inserted by cDNAs express cro- $\beta$-galactosidase hybrid protein in inclusion bodies, the low solubility of which protects the products against proteolysis. Moreover, the products of deletion mutants have naturally diverse molecular weights. Since the non-recombinant pEX product, $\beta$-galactosidase, has a much larger molecular weight than cDNA products, various deletion mutants are able to be analyzed under nearly the same condition without considering the difference in molecular weights.

Epitope mapping of the recombinant human SS-B/La protein has been reported by several investigators. Sturgess et al. 
A

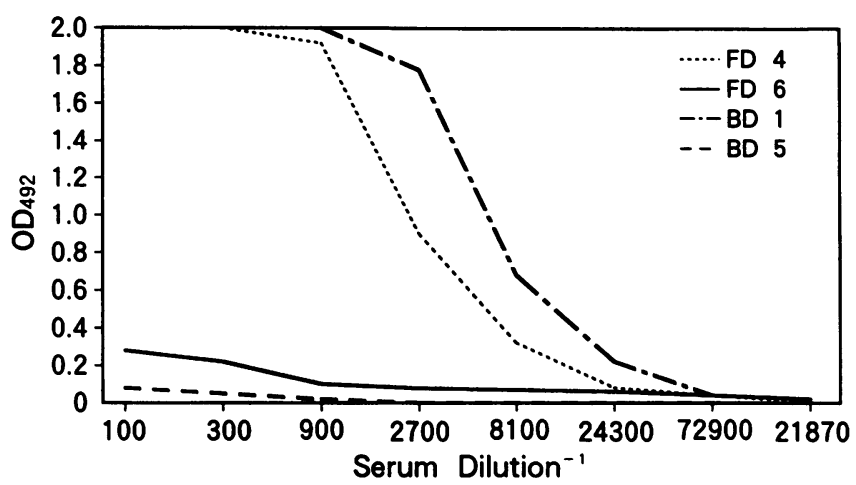

B

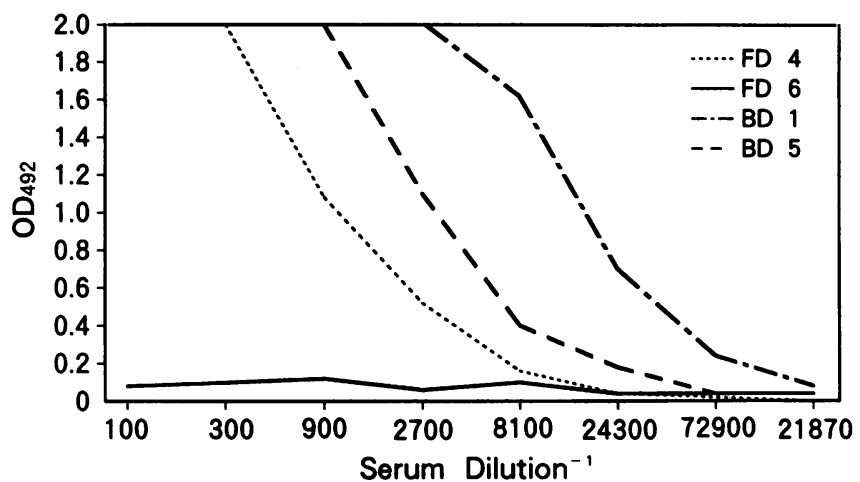

Figure 5. Quantitative analysis of anti-SS-B/La antibodies binding to the products encoded by deleted cDNAs. Multititer wells were coated with fusion proteins produced by recombinant $\mathrm{pEXs}$ (FD4, FD6, BD1, BD5). Sera from patients proved to have anti-SS-B/La antibodies were diluted with $1 \%$ skim milk at $1: 100$ to $1: 3^{7} \times 100$ and applied to the wells. The final data were calculated by subtraction of the value against the $\mathrm{pEX}$ protein from that against recombinant proteins. Sera from patients $1(A)$ and $2(B)$ reacted potently to the proteins produced by FD4 and BD1, while neither reacted to FD6. However, serum from patient 2 still reacted to BD5 less intensively than to BD1.

(14), using cDNA encoding a part of the SS-B/La protein, reported the 103 amino acid $\mathrm{COOH}$-terminal fragment as the only autoepitope. They failed to find antigenicity in Ept 1 and the region of Ept3. They did not succeed in expressing any of the $5^{\prime}$ parts of cDNA fragments and did not ensure the expression of the protein in the correct translational frame. Rauh et al. (15) used a non-full-length cDNA divided into many fragments by various restriction enzymes. They also used the pEX vector system for expression. They revealed at least three segments (amino acids 224-292, 293-335, 336-380) on the SS-B/ La protein that contained autoepitopes. This result is compatible with ours, although their assignments were rather broad and, most importantly, their cDNA did not cover the Ept1 segment. St. Clair et al. (16) used three non-overlapping cDNAs covering almost the whole protein coding region and expressed them in the $\lambda \mathrm{gt} 11 \mathrm{system}$. They reported that antigenic sites resided in a middle fragment (amino acid 111-242) of the SS-B/La protein. It was the first study in which a nearly full-length cDNA had been engaged. However, there are some
Table II. Antibodies Binding against the Hybrid Proteins Expressed by FD4, FD6, BD1, and BD5

\begin{tabular}{|c|c|c|c|c|}
\hline \multirow[b]{2}{*}{ Patient No. } & \multicolumn{4}{|c|}{ Binding units } \\
\hline & FD4 & FD6 & BD1 & BD5 \\
\hline 1 & 2,400 & $<100$ & 6,000 & $<100$ \\
\hline 2 & 1,200 & $<100$ & 16,800 & 2,900 \\
\hline 3 & 110 & $<100$ & 1,800 & $<100$ \\
\hline 4 & 7,300 & $<100$ & 35,000 & 6,900 \\
\hline 5 & 9,900 & $<100$ & 34,500 & 1,400 \\
\hline 6 & 930 & $<100$ & 3,100 & 190 \\
\hline 7 & 6,400 & $<100$ & 17,400 & 960 \\
\hline 8 & 1,900 & $<100$ & 6,900 & 3,300 \\
\hline 9 & 930 & $<100$ & 2,100 & 690 \\
\hline 10 & 130 & $<100$ & 220 & $<100$ \\
\hline 11 & 2,200 & $<100$ & 6,400 & 730 \\
\hline 12 & 5,400 & $<100$ & 48,100 & 14,000 \\
\hline 13 & 150 & $<100$ & 1,000 & 320 \\
\hline 14 & 550 & $<100$ & 1,000 & 800 \\
\hline 15 & 580 & $<100$ & 2,400 & $<100$ \\
\hline 16 & 100 & $<100$ & 580 & 150 \\
\hline 17 & 2,500 & $<100$ & 14,000 & 7,800 \\
\hline 18 & 1,600 & $<100$ & 3,800 & $<100$ \\
\hline 19 & 1,800 & $<100$ & 420 & 410 \\
\hline 20 & 200 & $<100$ & 3,700 & 1,600 \\
\hline 21 & 2,500 & $<100$ & 1,100 & 260 \\
\hline 22 & 100 & $<100$ & 1,100 & 390 \\
\hline 23 & 2,800 & $<100$ & 17,100 & 12,800 \\
\hline 24 & 100 & $<100$ & 100 & $<100$ \\
\hline 25 & 230 & $<100$ & 600 & 150 \\
\hline 26 & 3,000 & $<100$ & 11,500 & 6,400 \\
\hline 27 & 1,000 & $<100$ & 4,900 & 2,600 \\
\hline 28 & 270 & $<100$ & 860 & 500 \\
\hline Healthy donors & $<100$ & $<100$ & $<100$ & $<100$ \\
\hline
\end{tabular}

28 sera from patients with precipitating anti-SS-B/La autoantibodies were assayed by ELISA. Binding units are defined as the reciprocal of the serum dilution producing an $\mathrm{OD}_{492}$ of 1.0. Sera from healthy donors did not react with the recombinant autoantigens. Less than 100 binding units indicates an eventual lack of binding, i.e., $\mathrm{OD}_{492}$ values were below 0.3 in 1:100 dilution.

questionable points. First, the hybrid protein, reported to contain the amino acid 1-107 fragment, had a higher molecular weight in polyacrylamide/SDS gels than those containing longer segments of the SS-B/La protein. Next, in their ELISA assessment, the amino acid 111-408 fragment was more anti-

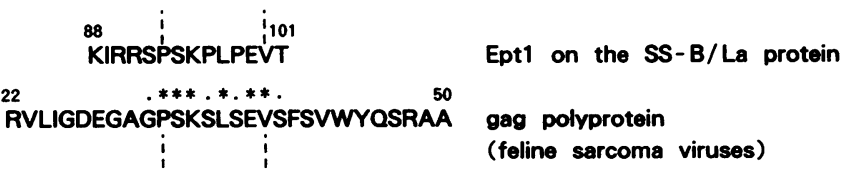

Figure 6. Homologous amino acid sequence of Ept1 on the SS-B/La protein and feline sarcoma virus gag polyprotein. Upper sequence represents amino acid 88-101 in Eptl on the SS-B/La protein. Lower sequence represents amino acid 22-50 in the gag polyprotein. Asterisks indicate amino acids that are identical in the two peptides. One dot indicates those that are analogous in their biochemical characteristics. 
genic with patients' sera than the 111-242 fragment, while the 242-408 fragment had little antigenicity. As we described before, $\lambda \mathrm{gt} 11$ with cDNA in incorrect translational frames could produce small amounts of proteins with unexpectedly high molecular weights even if the induced major hybrid proteins with lower molecular weights had no antigenicity. Therefore it is possible that the amino acid 1-107 and 242-408 fragments described in their paper were produced by expression in incorrect translational frames. The different assessment of Ept 1 and Ept2, located in amino acid 1-107 and 242-408 fragments, respectively, might be resulted from the incorrect expression or from different population of the patient sera.

Comparison of Ept1, Ept2, and Ept3 sequences with reported amino acid sequences in databases disclosed a high degree of homology between the amino acid 93-100, the $\mathrm{COOH}$-terminal part of Ept1, and the amino acid 32-39 of feline sarcoma virus gag polyprotein. Between unrelated proteins, the probability of matching six amino acids from eight amino acids is calculated to be $4.4 \times 10^{-7}$. Moreover, two different amino acids within this homologous region and flanking amino acids are conservative changes. The probability of having such changes is extremely low. The amino acid 32-39 resides in the leader peptide of the gag polyprotein. Besides, as we discussed, the COOH-terminal part of Ept 1 seemed to be the antigenic site. It is likely that the autoepitope on Eptl has actual homology to the gag polyprotein of the viruses or similar unknown viruses. Retrovirus infections are known to cause a broad range of chronic systemic diseases. They can be associated with oncogenesis or the pathogenesis of adult immunodeficiency syndrome. Quary and Keene (30) reported previously that part of the 70-kD protein of U1 snRNP was homologous to retroviral $\mathrm{p} 30^{\mathrm{gag}}$ protein and they were immunologically cross-reactive. Maul et al. (31) recently reported that one epitope on another autoantigen, human DNA topoisomerase I was homologous to the other region of retrovirus $\mathrm{p} 30^{\mathrm{gag}}$ protein. Even though the homologous epitopes have not shown to be the most distinct epitope, these reports suggested that retroviral infection might cause autoimmunity against some nucleoproteins and play a role on pathogenesis of autoimmune diseases. In Sjögren's syndrome, environmental factors, especially viral infections have been thought to play an important role in the initiation of the disease (32-34). In our study, we demonstrated that the region containing peptide homologous to a viral gag protein was universally recognized as the most distinct autoepitope. This leads us to the following speculations on the initiation of anti-SS-B/La autoimmunity. First, infection with exogenous retroviruses or expression of endogenous retroviruses that have structures similar to feline sarcoma viruses evoke an immune response against their gag proteins. Next, a certain expanded B cell repertoire against the gag protein could react with Ept 1 on the SS-B/La protein via cross-reactivity, and these B cells could present a part of the SS-B/La molecule to a host immune system as an immunogenic peptide, as suggested by Lanzavecchia (35). Alternatively, considering the nature of a leader protein which is likely to be within the cells, this homologous region could elicit $\mathrm{T}$ cell responses against the self SS-B/La protein. Thereafter the other parts of the SS-B/La protein with potential immunogenicity might begin to be recognized. Since antibody production against these additional epitopes seems to be defined not only by the biochemical character of the peptide but also the genetic background of the individual, they are not necessarily recog- nized equally by all patients' sera. Indeed, reactivity against the epitopes located on Ept 2 and Ept 3 varied according to the individual patient's serum, while Ept 1 was reactive with all sera. Efforts to examine the cross-reactivity of the gag protein with the Ept 1 peptide are in progress.

However, these evaluations should be carefully performed. Synthetic oligopeptides do not always represent the antigenicities of the real molecules. In addition, we suspect that feline sarcoma virus might not be the casative agent of Sjögren's syndrome. Another unknown virus having a similar structure could be the candidate. Thus we are now trying to express the large region of the gag leader protein in $E$. coli and to exchange some amino acids by site directed mutagenesis.

\section{Acknowledgments}

We would like to thank Dr. Susumu Sugai for supplying anti-SS-B/La antibody positive sera from his patients. We also appreciate the excellent technical assistance of Miss Hiroko Ichijo.

These studies were supported in part by grants from the Ministry of Health and Welfare and the Ministry of Education, Japan.

\section{References}

1. Tan, E. M. 1982. Autoantibodies to nuclear antigens (ANA): their immunobiology and medicine. Adv. Immunol. 33:167-240.

2. Tan, E. M. 1989. Antinuclear antibodies: diagnostic markers for autoimmune diseases and probes for cell biology. Adv. Immunol. 44:93-151.

3. Hang, L., J. H. Slack, C. Amundson, S. Izui, A. N. Theofilopoulos, and F. Dixon. 1983. Induction of murine autoimmune disease by chronic polyclonal B cell activation. J. Exp. Med. 157:874-883.

4. Tan, E. M., E. K. L. Chan, K. F. Sullivan, and R. L. Rubin. 1988. Antinuclear antibodies (ANAs): diagnostically specific immune markers and clues toward the understanding of systemic autoimmunity. Clin. Immunol. Immunopathol. 47:121-141.

5. Oldstone, M. B. A. 1987. Molecular mimicry and autoimmune disease. Cell. 50:819-820.

6. Harley, J. B., E. L. Alexander, W. B. Bias, O. F. Fox, T. T. Provost, M. Rejchlin, H. Yamagata, and F. C. Arnett. 1986. AntiRo(SS-B) and anti-La (SS-B) in patients with Sjogren's syndrome. $A r$ thritis Rheum. 29:196-206.

7. Rinke, J., and J. A. Steitz. 1982. Precursor molecules of both human 5 S ribosomal RNA and transfer RNAs are bound by a cellular protein reactive with anti-La lupus antibodies. Cell. 29:149-159.

8. Lerner, M. R., J. A. Boyle, J. A. Hardin, and J. A. Steitz. 1981 Two novel class of small ribonucleoproteins detected by antibodies associated with lupus erythematosus. Science (Wash. DC.) 211:400402.

9. Lerner, M. R., N. C. Andrews, G. Miller, and J. A. Steitz. 1981 Two small RNAs encoded by Epstein-Barr virus and complexed with protein are precipitated by antibodies from patients with systemic lupus erythematosus. Proc. Natl. Acad. Sci. USA. 78:805-809.

10. Kurilla, M. G., and J. D. Keene. 1983. The leader RNA of vesicular stomatitis virus is bound by a cellular protein reactive with anti-La lupus antibodies. Cell. 34:837-845.

11. Wilusz, J., and J. D. Keene. 1984. Interactions of plus and minus strand leader RNAs of the New Jersey serotype of vesicular stomatitis virus with the cellular La protein. Virology. 135:65-73.

12. Keene, J. D., S. L. Duetscher, D. J. Kenan, and A. Kelekar. 1987. Nature of the La and Ro RNPs. Mol. Biol. Rep. 12:235-238.

13. Chan, E. K. L., A. Francoeur, and E. M. Tan. 1986. Epitopes, structural domains, and asymmetry of amino acid residues in SS-B/La nuclear protein. J. Immunol. 136:3744-3749.

14. Sturgess, A. D., M. G. Peterson, L. J. McNeilage, S. Whittingham, and R. L. Coppel. 1988. Characteristics and epitope mapping of a cloned human autoantigen La. J. Immunol. 140:3212-3218. 
15. Rauh, A. J. G., H. Hornig, and R. Luhrmann. 1988. At least three distinct $B$ cell epitopes reside in the C-terminal half of La protein, as determined by a recombinant DNA approach. Eur. J. Immunol. 18:2049-2057.

16. St. Clair, E. W., D. S. Pisetsky, C. F. Reich, and J. D. Keene 1988. Analysis of autoantibody binding to different regions of the human La antigen expressed in recombinant fusion proteins. J. Immunol. 141:4173-4180.

17. Chambers, J. C., D. Kenan, B. J. Martin, and J. D. Keene. 1988. Genomic structure and amino acid sequence domains of the human La autoantigen. J. Biol. Chem. 263:18043-18051.

18. Yamamoto, K., H. Miura, Y. Moroi, S. Yoshinoya, M. Goto, K. Nishioka, and T. Miyamoto. 1988. Isolation and characterization of a complementary DNA expressing human U1 small nuclear ribonucleoprotein C polypeptide. J. Immunol. 140:311-317.

19. Kohsaka, H., K. Yamamoto, H. Fujii, N. Miyasaka, H. Miura, Y. Tanaka, K. Nishioka, and T. Miyamoto. 1989. Molecular cloning of cDNAs expressing SS-B/La protein. J. Autoimmunity. 2(Suppl.):353-357.

20. Young, R. A., and R. W. Davis. 1983. Yeast RNA polymerase II genes: Isolation with antibody probes. Science (Wash. DC). 222:778-782.

21. Stanley, K. K., and J. P. Luzio. 1984. Construction of a new family of high efficiency bacterial expression vectors: identification of cDNA clones coding for human liver proteins. EMBO (Eur. Mol. Biol. Organ.) J. 3:1429-1434.

22. Okayama, H., and P. Berg. 1983. A cDNA cloning vector that permits expression of cDNA inserts in mammalian cells. Mol. Cell. Biol. 3:280-289.

23. Olmsted, J. B. 1981. Affinity purification of antibodies from diazotized paper blots of heterogenous protein samples. J. Biol. Chem. 256:11955-11957.

24. Henikoff, S. 1984. Unidirectional digestion with exonuclease III creates targeted breakpoints for DNA sequencing. 1984. Gene. 28:351-359.
25. Putney, S. D., S. J. Benkovic, and P. R. Schimmel. 1981. A DNA fragment with an $\alpha$-phosphorothioate nucleotide at one end is asymmetrically blocked from digestion by exonuclease III and can be replicated in vivo. Proc. Natl. Acad. Sci. USA. 78:7350-7354.

26. Sanger, F. 1981. Determination of nucleotide sequence in DNA. Science (Wash. DC). 214:1205-1210.

27. Chan, E. K. L., K. F. Sullivan, and E. M. Tan. 1989. Ribonucleoprotein SS-B/La belongs to a protein family with consensus sequence for RNA-binding. Nucleic Acids Res. 17:2233-2244.

28. Besmer, P., J. E. Murphy, P. C. George, F. Qiu, P. J. Bergold, L. Ledermen, H. W. Snyder, Jr., D. Brodeur, E. E. Zuckerman, and W. D. Hardy. 1986. A new acute transforming feline retrovirus and relationship of its oncogene v-kit with the protein kinase gene family. Nature (Lond.). 320:415-420.

29. Hampe, A., M. Gobet, J. Even, C. J. Sherr, and F. Galibert. 1983. Nucleotide sequences of feline sarcoma virus long terminal repeats and $5^{\prime}$ leaders show extensive homology to those of other mammalian retroviruses. J. Virol. 45:466-472.

30. Query, C. C., and J. D. Keene. 1987. A human autoimmune protein associated with U1 RNA contains a region of homology that is cross-reactive with retroviral p30 $30^{2 a 8}$ antigen. Cell. 51:211-220.

31. Maul, G. G., S. A. Jimenez, E. Riggs, and D. Ziemnicka-Kotula. 1989. Determination of an epitope of the diffuse systemic sclerosis marker antigen DNA topoisomerase I. Proc. Natl. Acad. Sci. USA. 86:8492-8496.

32. Whittingham, S., J. McNeilage, and I. R. Mackay. 1985. Primary Sjögren's syndrome after infectious mononucleosis. Ann. Intern. Med. 102:490-493.

33. Yamaoka, K., N. Miyasaka, and K. Yamamoto. 1988. Possible involvement of Epstein-Barr virus in polyclonal B cell activation in Sjögren's syndrome. Arthritis \& Rheum. 31:1014-1021.

34. Green, J. E., S. H. Hinrichs, J. Vogel, and G. Jay. 1989. Exocrinopathy resembling Sjögren's syndrome in HTLV-1 tax transgenic mice. Nature (Lond.). 341:72-74.

35. Lanzavecchia, A. 1988. Clonal sketches of the immune response. EMBO (Eur. Mol. Biochem. Organ.) J. 7:2945-2951. 